\title{
Revisión del concepto
} de internal branding: la transmisión de la

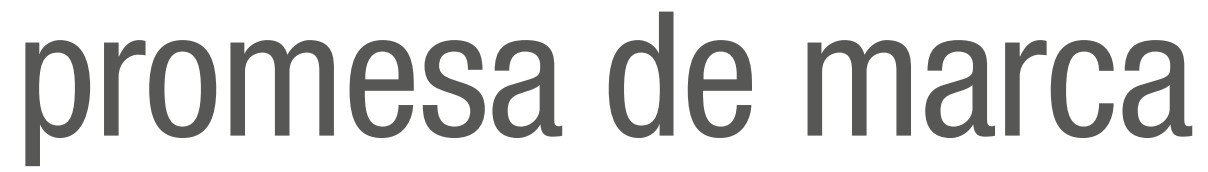

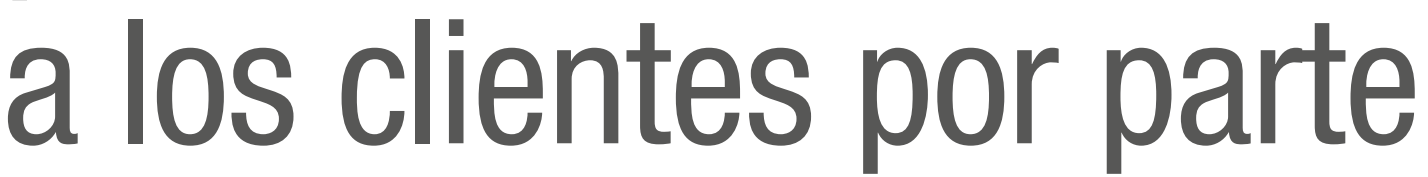
de los empleados

Carlos de la Guardia, ESIC, España; carlos.delaguardia@brandgineering. net; Ana $\mathrm{M}^{\mathrm{a}}$ Enrique, Universidad Autónoma de Barcelona, España; Anamaria.enrique@uab.cat

REsumen

Este artículo presenta una revisión del concepto de internal branding y una investigación de campo en base a quince entrevistas a profesionales y académicos de diferentes ámbitos relacionados con el objeto de estudio. Se concluye que el internal branding es una disciplina joven con escasa aplicación en las empresas españolas y evidencia la necesidad de fomentar que los empleados, activos estratégicos intangibles, aumenten su nivel de engagement con la organización para que contribuyan a mejorar la eficacia en la transmisión de la promesa de marca a los clientes.

Palabras clave

internal branding; marketing; empleados; clientes; marca; organización

Revision of internal branding concept: the transmission of the brand promise to clients by employees

ABSTRACT

This article presents a revision of the internal branding concept as well as a primary research based on fifteen interviews to professionals and academics of different fields related to the subject of study. The conclusion points out that internal branding is a young discipline with little application to Spanish companies and it points out the need to foster employee engagement, since they are intangible strategic assets, to improve the deliverance of the promise of the brand to clients

Keywords

internal branding; marketing; employees; clients; brand; organization 


\section{introducción y objeto de estudio}

El propósito de este artículo es analizar desde un punto de vista teórico y práctico la implementación de los programas de internal branding en las organizaciones españolas. Se ha detectado una carencia de investigaciones en el ámbito de internal branding, sobre todo en lengua hispana, lo cual va unido a su incipiente aplicación en las empresas del territorio español. La disciplina de internal branding se debe enmarcar dentro del marketing interno y de las políticas de comunicaciones integradas de marketing (IMC) y tiene como fin fundamental desarrollar programas dirigidos a los empleados de una organización para que mejoren la comprensión de la promesa de la marca y así influenciar en su actitud y su comportamiento ante los clientes de la organización.

La marca, a través de sus acciones comunicacionales, crea una serie de expectativas a sus clientes en base a su promesa y se pretende que cada vez que entren en contacto con la marca la experiencia resultante se enmarque dentro de estas promesas para que la marca pueda desplegar su competitividad. Que la marca cumpla su promesa es fundamental para que pueda desarrollar la relación Marca/Cliente y promover el sentimiento de satisfacción. La valoración positiva de la promesa de la marca por sus clientes es la base sobre la que se asienta la construcción de una relación mutuamente beneficiosa que consolide el sentimiento de fidelidad.

Para que la promesa de la marca despliegue todo su potencial, es necesario que se identifiquen y se gestionen diversos factores: la gestión del conocimiento, la comprensión, la experiencia con el producto o servicio, el grado de afinidad y de compromiso del empleado con la marca, etc. Todos estos factores contribuyen activamente en la transmisión de la promesa de marca que, a su vez, contribuyen a la creación de experiencias totales positivas en los clientes. Esta transmisión se realiza en cada uno de los puntos de contacto de los clientes con la marca y es especialmente importante cuando son los empleados los que interactúan con ellos.

Por lo tanto, la contribución de esta investigación radica en establecer cómo es percibido el rol del empleado en la gestión de la marca en las organizaciones, cómo se gestionan los programas de internal branding y qué departamentos de la organización son los responsables idóneos para articular estos programas. Para ello, este trabajo contribuye, en primer lugar, a realizar una aproximación teórica al concepto de internal branding $y$, en segundo lugar, aporta las valoraciones de un conjunto profesionales y académicos de diferentes ámbitos convergentes en el diseño, la aplicación y la evaluación de programas de internal branding en las organizaciones españolas.

\section{Fundamentación teórica}

La literatura moderna existente en torno al fenómeno del marketing argumenta que se está produciendo un cambio en el paradigma tradicional - hasta ahora centrado en la lógica del marketing de producto - hacia una orientación centrada en los consumidores (Lambin 2012; King \& Grace, 2012). Poner al consumidor o al cliente en el centro del modelo de negocio implica profundizar en su conocimiento, con el mayor grado de detalle posible, para que la marca pueda comprender mejor, satisfacer mejor y con mayor intensidad sus deseos (Keller, 2003). En su evolución, el marketing ha ido desarrollando especialidades, metodologías y herramientas específicas como el marketing experiencial, el marketing relacional o el internal branding, para ir abordando las adaptaciones requeridas por el entorno competitivo y forzar a la vez la evolución organizacional. El internal branding es una nueva disciplina basada en la gestión activa del rol de los empleados en la entrega de la promesa de la marca para conseguir que los clientes tengan unas experiencias positivas totales con la marca y que se traduzca en una mayor fidelidad del consumidor con la marca (Berry, 2000; Grace \& O'Cass, 2005; Schmitt, 2011; Schultz et al., 2003). El internal branding nace con el objetivo de mejorar la relación Empleado/Marca para que así mejore la relación Cliente/Marca (Sartain \& Schumann, 2006).

En el actual entorno competitivo, las empresas tratan de facilitar las condiciones idóneas para que el contacto entre la empresa y el cliente sea continuo, satisfactorio y tenga el significado que los gestores de la marca desean (Schultz et al., 2003; Punjaisri \& Wilson, 2011). Para ello, las empresas analizan cómo es esta experiencia y cómo la marca puede sobrepasar las expectativas del cliente (Aaker, 2002). El objetivo es establecer una relación duradera y satisfactoria para todas las partes involucradas (Keller, 2003). Y de aquí surge la imperante necesidad de que los empleados estén involucrados y comprometidos emocionalmente con la marca, es decir, que se genere engagement ${ }^{1}$ para que el cliente reciba la promesa de la marca de acuerdo con sus expectativas y sus experiencias pre-

1. El engagement es definido por Welch (2011:341) como "un estado psicológico dinámico e influenciable que vincula al empleado con la organización que se manifiesta en el desempeño individual de los miembros de la organización en términos físicos, cognitivos y emocionales y que es influenciado por las comunicaciones internas de la organización". 
vias (Welch, 2011). Sin gestionar efectivamente el engagement de los empleados con la marca no se puede planificar ni gestionar la calidad y la eficacia de la transmisión de la promesa de la marca a los clientes. (Welch, 2011). Si la organización no planifica ni gestiona cómo aumentar el engagement de los empleados, ésta no estará aprovechando las oportunidades que cada interacción produce entre el cliente y la marca para aumentar y consolidar su fidelidad (Thomson \& De Chernatony, 1999; Sartain \& Schumann, 2006; Punjaisri \& Wilson, 2011).

La especialidad del internal branding nace bajo la necesidad de que las organizaciones profundicen, cada vez que hay una interacción, en el desarrollo del sentimiento de fidelidad hacia la propuesta de valor que la marca hace a sus diferentes públicos por medio del cumplimiento de las expectativas que la organización ha creado en ellos (Manhert, 2009). En cada interacción la marca revalida la competitividad de su promesa reafirmando así el comportamiento fiel de los clientes. La marca por medio de sus comunicaciones de marketing crea unas expectativas ante sus clientes externos, invirtiéndose enormes cantidades de recursos (Schultz et al., 2003), para promover la promesa que la marca hace a su mercado. La transmisión de la promesa de la marca genera en sus audiencias unas expectativas determinadas que pretenden crear unas experiencias únicas que conecten a la marca con los clientes, conocido como el external branding. A medida que los actores que compiten en un mercado refuerzan sus promesas complementando sus ofertas tangibles con beneficios intangibles en forma de servicios, garantías, etc, los empleados se convierten en transmisores activos de la promesa de la marca (Costa, 2012).

El internal branding es una disciplina muy joven y justo ahora comienza a despertar el interés de las organizaciones y de los académicos. Es por ello, que todavía existen muchas lagunas conceptuales, teóricas y metodológicas (Mortimer 2002; Foster et al., 2010). Es importante evidenciar una de estas lagunas conceptuales pues es motivo de confusión en la literatura: no se debe confundir el employer branding con el internal branding (Zucker, 2002). El primero, son los programas que la organización hace para captar empleados en un mercado laboral de demanda. Es cómo se presenta la organización ante los mercados laborales para atraer a los perfiles adecuados (Aguado \& Jiménez, 2009). Por otro lado, el internal branding, es la disciplina que desarrolla la lealtad en la relación Empleado/Marca y que, conjuntamente con la colaboración del departamento de $\mathrm{RR} \mathrm{HH}$, tiene como objetivo retener y aumentar el nivel de engagement de los empleados actuales (Sartain \& Schuman, 2006; Punjaisri \& Wilson, 2011; MacLaverty \& Oddie, 2015). La finalidad de los programas de internal branding es definir cómo debe ser la interacción entre los empleados y la marca para generarle al cliente una experiencia total positiva con la marca, y por ende, con la organización, y que estimule y refuerce la percepción, la voluntad y el comportamiento de sus clientes (King \& Grace, 2008; Mahnert \& Torres, 2007; Sartain \& Schumann, 2006; Foster et al., 2010). Es decir, es planificar cómo debe ser la relación Empleado/ Marca para que repercuta positivamente en la relación Cliente/Marca (Ind, 2004). Según, Punjaisri, Wilson, et al (2009: 209-226) internal branding se define como "la herramienta que se asegura que los mensajes de la marca (la promesa de la marca) sean transformados por los empleados en una realidad que refleje las expectativas de la experiencia de los clientes con la marca".

Por tanto, debemos considerar al internal branding una práctica multidisciplinaria que requiere equipos de trabajo transversales, fuertemente apoyados y liderados por la Dirección, para diseñar sus programas y para que la organización los asuma, invierta en ellos consistentemente y los implemente adecuadamente (MacLaverty \& Oddie, 2015). Estos programas otorgan a los empleados la capacidad de vivir la marca en el acto de desarrollar su trabajo puesto que trabajan sobre el conocimiento y compresión de la promesa de la marca, sobre la afinidad entre los valores de ambos, y sobre la motivación y comportamiento de los empleados con la marca y sus públicos (Punjaisri, Wilson et al, 2009; Punjaisri \& Wilson, 2011). La lealtad y compromiso de los empleados hacia la marca - partes esenciales del engagement - se consiguen por medio de un esfuerzo continuo, consistente e inspirador para que ellos produzcan la misma experiencia total positiva que la marca les promete a los clientes (Albrecht, 2010). Dicho esto, apuntar que es responsabilidad de los gestores de la marca facilitar que el empleado pueda y quiera involucrarse con la marca (Ind, 2001). Como es obvio, el internal branding solamente es eficiente si la relación Empleado/Organización es estable, satisfactoria y si los niveles de confianza son los adecuados, por lo que la involucración de $\mathrm{RRHH}$ y de la Dirección es absolutamente necesaria.

Los programas de internal branding están orientados a conseguir que el empleado no solo preste un servicio de la mejor calidad posible de acuerdo con la promesa de la marca, si no que aspira a promover el rol de embajadores de la marca que repre- 
sentan porque se sienten afines y comprometidos con la reputación que tiene (Ind, 2004; Gillis, 2011).

Por último, incidir en que el concepto de internal branding, a pesar de ser reciente, está sufriendo constantes evoluciones porque genera interés entre los gestores de la marca. Ravens (2014) en su obra Internal Brand Management in international contexts hace un repaso exhaustivo a las diferentes escuelas que han definido los diferentes estadios de evolución de esta disciplina y ha evaluado los diferentes modelos propuestos por diversos autores.

\section{Metodología}

En base a la teoría descrita nos planteamos la siguiente pregunta general de investigación: ¿cómo los programas de internal branding pueden contribuir a generar engagement entre los empleados y la marca con el fin de consolidar la fidelidad de los clientes/consumidores hacia la marca? Es decir, cómo influyen los programas de internal branding en la relación Empleado/Marca y cómo se aplica en las empresas españolas.

La investigación realizada es de carácter cualitativo basado en la técnica de la entrevista enfocada. La elección de este tipo de entrevistas es idónea cuando de antemano está preestablecido un tema de interés hacia el que se va a orientar la conversación y a partir del cual se ha seleccionado a la persona que será objeto de la entrevista (Galindo, 1998). Este método nos ha permitido interpretar las opiniones y puntos de vista de un grupo de sujetos especialistas de diferentes ámbitos convergentes con la disciplina de internal branding y, de este modo, aproximarnos en la praxis al actual estado de la cuestión. El diseño de la muestra se ha realizado en una única fase y se ha utilizado lo que Wimmer y Dominick (1996) llaman el muestreo estratégico, que se basa en la selección de fuentes primarias en base a los propios criterios del investigador. Este tipo de muestreo se utiliza en numerosas ocasiones cuando se trata de un estudio cualitativo con una muestra reducida donde se busca profundizar en la información por encima de la estadística. Por lo tanto, y en base a estos criterios, la muestra se compone de quince sujetos de reconocido prestigio, por su carrera profesional o académica, que han sido agrupados por su área de conocimiento principal. Estos sujetos desempeñan su trabajo en organizaciones ubicadas en el territorio español. En este sentido, para la selección de los entrevistados se han utilizado los siguientes cinco filtros:

- Profesionales del Área de Marketing con responsabilidades gerenciales: esta muestra está formada por cinco profesionales que desem- peñen o hayan desempeñado en su carrera el cargo de directores de Marketing y que además hayan tenido responsabilidades ejecutivas sobre marcas y sobre equipos de trabajo.

- Profesionales del Área de Comunicación: esta muestra está formada por dos profesionales que desempeñen o hayan desempeñado funciones en su carrera en el cargo de directores de comunicación.

- Profesionales del Área de Recursos Humanos: esta muestra está formada por tres profesionales que desempeñen o hayan desempeñado en su carrera el cargo de directores de Recursos Humanos.

- Profesionales del Área de la comunicación de marca: esta muestra está formada por tres profesionales que tengan experiencia en la gestión y planificación de marcas.

- Profesionales del Ámbito Académico: en este ámbito se incluye a dos profesores e investigadores de los ámbitos relevantes próximos al internal branding.

\section{Desarrollo}

La captación de la muestra se realizó mediante previo contacto telefónico o telemático, dependiendo de la disponibilidad de cada uno de los entrevistados. El primer contacto se realizó a través de correo electrónico, el segundo vía telefónica y el tercer contacto fue la propia entrevista enfocada. Las entrevistas se realizan en los meses de junio y julio de 2014, de una hora y media aproximada de duración. A estos expertos se les sometió a un cuestionario abierto con el fin de conocer su visión y opinión sobre el tema. Las cuestiones abordadas giraron en torno a tres ejes centrales:

- El rol del empleado en la gestión de la marca.

- Conocimiento y experiencia en la gestión de programas de internal branding.

- La ubicación en la organización del equipo responsable de internal branding y su dependencia orgánica.

- Una vez elaboradas las transcripciones de las entrevistas, se realizó un análisis de contenido de cada una de ellas y se clasificó la información obtenida en función de las categorías de análisis establecidas.

\section{Resultados}

El rol del empleado en la gestión de la marca.

La información aportada por los entrevistados confirma lo expresado por los autores de referencia: el creciente interés de las organizaciones por el internal branding es un avance significativo en el compromiso de la organización de gestionar 
eficientemente la relación Empleado/Marca como estrategia para aumentar y consolidar la fidelidad de los grupos de interés de la organización con la marca. En este sentido, las aportaciones de los entrevistados son unánimes.

Se identifican las condiciones necesarias para que una organización pueda maximizar el retorno a la inversión en programas de internal branding. En primer lugar, los entrevistados coinciden en sus respuestas en esta cuestión enfocándose en la capacidad que las organizaciones tienen para gestionar los recursos humanos como activo estratégico intangible y, por lo tanto, como elemento fundamental en la capacidad que tienen de generar valor. Es necesario que la organización identifique y justifique la importancia del valor del factor humano y lo gestione profesionalmente para que las políticas de internal branding resulten positivas en la gestión de la satisfacción de los empleados y, por ende, del cliente. -En segundo lugar, la organización debe entender que el empleado es uno de los factores más importantes en la generación de valor al cliente, independientemente de dónde realicen su función, si de cara al cliente -front office- o no back office-. El empleado, independientemente del puesto que ocupe en la organización, con su comportamiento y actitud gestiona voluntariamente sus relaciones con el cliente de la marca y modula y cualifica las experiencias que la marca genera durante las interacciones. Por tanto, el empleado es quien hace tangible la estrategia de la marca y humaniza la promesa de la marca cara a los clientes.

Tanto los empleados como la marca son considerados, junto con los otros activos intangibles, activos estratégicos que componen y cualifican la ventaja competitiva de la marca y de la organización. Los empleados hacen competitiva y atractiva a la marca y son fuente esencial de diferenciación y relevancia sostenible.

Bajo estos planteamientos que ponen al empleado en el centro de la generación de valor, los entrevistados coinciden en que el rol del empleado en la gestión de la marca es múltiple:

- Son los transmisores de la promesa de la marca $y$, de hecho, su comportamiento y su actitud, entran a formar parte de ella.

- Son uno de los puntos de contacto más importantes en la relación Cliente/Marca y tienen una gran capacidad para influenciar -positiva o negativamente -en las experiencias que la marca genera. Son la humanización de la marca.

- Son un público objetivo prioritario y estratégico.

- Son los gestores del cambio en las organizaciones y en muchos casos son los que las potencian o las inhiben.
- Son prescriptores de la organización y la marca al resto de los públicos.

Independientemente de la necesidad de gestionar esta relación, todos los entrevistados opinaron que gestionar la relación Empleado/Marca es un área de mejora para la mayoría de las organizaciones y que existen ejemplos y modelos que demuestran que mejorar esta relación redunda en la mejora de la organización en su conjunto tanto a corto como a largo plazo. Igualmente, los entrevistados determinan como necesario que la gestión de la relación Empleado/Marca se sistematice y se planifique en las organizaciones y que es una fuente a explotar para incrementar la fidelidad de los públicos de la empresa en base a la gestión de la satisfacción que tienen con la marca. Aumentar la fidelidad de los grupos de interés supone en condiciones normales una mejora en el retorno de las inversiones hechas en la organización.

De las respuestas se deduce que existe una necesidad latente en las organizaciones de contemplar al empleado como cliente interno y de tratarlo con el mismo rigor y profesionalidad con el que se trata a los clientes externos. Del mismo modo que las organizaciones hacen grandes esfuerzos para ganarse la recomendación de clientes externos y consumidores, han de hacerlo hacia dentro de la organización.

En esta cuestión, los entrevistados mencionan repetidamente como referente el concepto de empleado como embajador de la marca como si esta condición fuese un objetivo aspiracional y casi utópico. Entienden la importancia que tiene para la marca y la organización que el empleado "vista la camiseta" de la organización pero sus respuestas inducen a pensar que es algo casi inalcanzable en las circunstancias actuales. El concepto de embajador de marca, sin embargo, produce un cierto rechazo en los entrevistados porque, semánticamente, lleva implícita una aparente intención homogeneizadora y uniformadora del comportamiento de los empleados que le resta credibilidad al comportamiento del empleado cara al cliente.

Los entrevistados reconocen que los programas orientados a alinear el comportamiento del empleado con los valores de la marca mejoran no solamente su rendimiento individual si no que, además, mejora la vinculación y la motivación a la organización. Todos los entrevistados reconocen que la satisfacción de los empleados mejora los resultados de la organización.

En conclusión, el internal branding nace precisamente para cubrir la necesidad de gestionar eficientemente la relación Empleado/Marca para que, en la visión de los entrevistados, se pueda 
maximizar la calidad de la interacción entre el cliente y la marca. El internal branding por lo tanto actúa como un rol facilitador de la relación entre producto/servicio/marca y sus grupos de interés.

\section{Gestión del internal branding en las organizaciones/marcas}

En este asunto llama la atención que la verbalización de las palabras internal branding no es reconocida por ninguno de los entrevistados per se aunque sí es claramente reconocida por su significado y admiten haber participado activamente en acciones de naturaleza similar al internal branding.

Una vez solucionado el inicial escollo semántico, todos los entrevistados coinciden en señalar que en la mayoría de las organizaciones es necesario gestionar más eficientemente la relación Empleado/Marca porque redunda en beneficios individuales y colectivos. Aportan ejemplos y experiencias concretas de acciones que tuvieron éxito y otras que fracasaron pero consolidaron su creencia en que gestionar la relación Empleado/ Marca es un área estratégica en las organizaciones y que requiere la evolución de los paradigmas actuales para generar nuevos enfoques.

Desde el enfoque de la orientación al cliente, todos los entrevistados consideran que cada vez es más importante y predominante el rol de los empleados en el proceso de fidelización de los clientes. A medida que las propuestas de la marca ganan peso frente a la funcionalidad, la relación Cliente/Marca se intensifica, y el rol del empleado es percibido por el cliente como la tangibilización de la promesa de la marca; son la parte más humana de la promesa de la marca y de las experiencias que ésta promueve. Por lo tanto, es deseable que la marca mantenga unos niveles altos de engagement con los empleados porque puede influir directa y positivamente en la calidad de relación Cliente/Marca. Es más, el internal branding también puede afectar positivamente en la relación Empleado/Organización y en las relaciones Empleado/Empleado, teniendo consecuencias positivas en la lealtad de los empleados actuales, en la atracción de talento a la organización (employer branding) y en una mejora generalizada de la productividad y del clima laboral.

Desde el punto de vista de la gestión de la marca, el internal branding también contribuye a que la percepción de la marca sea homogénea a través de todos sus públicos así como en los efectos positivos que esto conlleva tanto en la gestión de la comunicación de la marca como en la cuenta de explotación de la organización.

El internal branding es por lo tanto, según opinión unánime de los entrevistados, una disciplina integradora y transversal que afecta directamente a la capacidad de la organización de generar valor sostenible y, por ende, en el desarrollo de las ventajas competitivas que la organización necesita gestionar para competir en su mercado. Es por lo tanto una disciplina estratégica para la organización pues pone en valor gran parte de sus activos estratégicos intangibles. Sin embargo, admiten que estos programas son todavía escasos y, al ser programas internos no pueden facilitarnos toda la información.

Cuando se pregunta a los entrevistados por la ausencia de los programas de internal branding en las organizaciones sus respuestas son unánime y tienen los siguientes puntos en común:

a) Expresan cierta sorpresa porque el concepto básico de que "el empleado satisfecho es más productivo" aun siendo obvio no deja de ser más que un deseo, especialmente en la coyuntura española actual. Mientras que en las organizaciones multinacionales, ya sea bajo la denominación de internal branding o cualquier otra, ya tienen programas que aspiran a aumentar el engagement con la organización y con sus marcas, la falta casi total de estos programas en las pymes es un área evidente de mejora.

b) Los entrevistados creen que el nivel de engagement del empleado con la marca es percibido como parte del patrimonio de la marca y como tal, si es gestionado adecuadamente, será parte activa de su ventaja competitiva. Por lo que se estima necesario que las organizaciones asuman que el empleado es una variable muy importante en la trasmisión de la promesa de la marca al cliente y que es necesario que los empleados estén emocionalmente cerca de la marca porque genera actitudes y comportamientos que refuerzan la relación Marca/Cliente.

c) La aportación de valor de la relación Empleado/ Marca es solamente intuida en las organizaciones pero no es cuantificada para ser medida y evaluada. Esto dificulta en gran medida la elaboración de estrategias específicas y la dotación de recursos para su ejecución. Se apunta que sería necesario desarrollar sistemas de medición de "variables blandas" y poder determinar su aportación de valor a la organización. Dentro de los límites de cuantificación de los fenómenos en las Ciencias Sociales, se prevé que, ante la creciente necesidad de gestionar el engagement de los empleados con la marca y/u organización, se puedan desarrollar metodologías y procesos de cuantificación de esta aportación de valor para que los accionistas respalden a los financieros en la aprobación de las inversiones necesarias. 
d) Especifican que la comunicación de la marca a sus clientes internos es una responsabilidad no asumida por ningún departamento (excepto en las situaciones de "marca organización") siendo el departamento de marketing el único que tiene un interés directo en el tema. Esta función no suele existir en las organizaciones, excepto en el sector servicios por la coincidencia entre la marca y el naming de la organización. En el caso de las empresas del sector servicios y que sean monomarca, la responsabilidad de la comunicación de la "marca organización" recae sobre el departamento de comunicación, en el caso de que tengan uno. Por otro lado, la comunicación interna como herramienta del departamento de RR HH no tiene como función comunicar la marca a los empleados. Este vacío en la organización conlleva además una falta de la asignación de recursos para la definición, implementación y ejecución de los programas de internal branding.

e) Consideran que la comunicación interna debe evolucionar hacia la gestión de conversaciones más que transmitir información funcional y administrativa. De hecho, se evidencia la necesidad de que la disciplina de RR HH en sí misma se adapte a los nuevos paradigmas de la economía intangible.

Una vez más, todos los entrevistados están de acuerdo en que la gestión de esta relación implica colocar al empleado en uno de los centros estratégicos de interés para la marca, junto con el cliente. Es, por lo tanto necesario tratar a los empleados como un cliente interno y aplicarle las mismas metodologías, procesos y herramientas de análisis que se aplican regularmente al cliente desde el departamento de marketing.

El internal branding es, por tanto, parte de la respuesta a esta necesidad de reorientación de las organizaciones hacia al cliente en vez de hacia el producto porque se centra en modular la calidad de las relaciones Cliente/Marca a través de la gestión de la relación Empleado/Marca. El precepto básico del internal branding es: empleados satisfechos, clientes satisfechos.

\section{Departamentos de la organización (actores) directamente responsables en la creación y gestión de los programas de internal branding} Todos los entrevistados están de acuerdo en que los empleados son esenciales para la organización y que deben ser tratados igualmente por el internal branding. Los entrevistados entienden que los programas de internal branding son transversales a toda la organización y que todos los empleados deben ser considerados como parte del público objetivo, independientemente de su posición y funciones en la compañía. Con respecto a los departamentos de la organización que deben estar involucrados en la formulación, elaboración, implementación y evaluación de los programas de internal branding, se presentan los que más han sido nombrados por los entrevistados:

a) El departamento de $\mathrm{RR} H \mathrm{H}$ como último responsable de diseñar las políticas que gestionen la calidad de la relación Empleado/Organización, es decir, orientadas a la gestión del engagement.

b) El departamento de marketing como responsable de formular la estrategia de la marca y los planes de marketing a los clientes externos. Este departamento tiene la experiencia y las herramientas para comunicar eficientemente con los públicos objetivos de la marca. Entre otras habilidades, es relevante evidenciar que este departamento es experto en la planificación estratégica de la comunicación de marca como herramienta imprescindible para mejorar la- eficiencia comunicativa de la marca.

c) Especialmente en el caso de que la organización sea del sector servicios, donde la interacción Cliente/Empleado es directa e intensa, también se tendría que incluir al departamento de operaciones como susceptible de estar involucrado en los programas de internal branding.

d) A pesar de que cada organización adapta los protocolos y los procesos de sus programas de internal branding a sus características organizacionales, hay consenso en que, en el caso de que la organización cuente con un departamento de comunicación, éste debería ser el coordinador y garante de los programas de internal branding. El Director de comunicación sería el responsable de que la marca fuese percibida homogéneamente ante todos los públicos de la organización, con la implicación activa del departamento de marketing, $\mathrm{RR} \mathrm{HH}$, operaciones y/u otros departamentos relevantes porque asegura que la comunicación y, por ende, la percepción de la marca sea homogénea interna y externamente.

\section{Conclusiones}

En entornos competitivos, las organizaciones necesitan extraer todo el valor posible de sus activos para mantener sus ventajas competitivas. Las organizaciones cuentan con los activos estratégicos intangibles que deben ser gestionados con la comprensión, el rigor y el escrutinio al que son sometidos los activos tangibles. Los empleados, las marcas y la comunicación son tres activos in- 
tangibles con los que trata el internal branding El marketing, como disciplina que promueve la adaptación de las organizaciones a los cambios de los mercados, propone enfocar esos conocimientos, esas metodologías, procesos y herramientas utilizados en el análisis y evaluación de audiencias externas hacia los empleados como fuente de valor. Para ello, se desarrolla la disciplina del internal branding, para gestionar el nuevo entorno centrado en la gestión de relaciones, en este caso la relación Cliente/Marca a través de la gestión de la relación Empleado/Marca.

Es una disciplina joven, poco conocida y que, al ser una herramienta de mejora interna de las organizaciones, las evidencias y los ejemplos son escasos, especialmente, en el ámbito no anglosajón.

En esta investigación se ha pretendido estudiar el concepto de internal branding a partir de fuentes secundarias y también articulando un estudio de campo. Se han realizado una serie de entrevistas enfocadas a una muestra de especialistas competentes en el área de marketing, comunicación o $\mathrm{RRHH}$, áreas que convergen con la materia de internal branding, con el objetivo de ratificar los aprendizajes de la investigación documental y para ubicarla en la realidad de las organizaciones que operan el mercado español.

Podemos decir que el internal branding es una disciplina necesaria para la mejora de las relaciones Cliente/Marca. Es la voluntad de gestionar la interacción de la marca con los empleados en un determinado entorno y momento, y ante unas determinadas expectativas. El propósito de esta interacción es que la promesa de la marca sea transmitida por los empleados eficientemente para que la relación Cliente/Marca sea lo más estable y beneficiosa para todas las partes.

El internal branding pretende actuar sobre el comportamiento de los empleados. Las organizaciones necesitan fomentar que los empleados aumenten su nivel de engagement con la marca y con la organización porque la satisfacción produce beneficios sostenibles. Los empleados son activos estratégicos intangibles, parte esencial de la organización y son los transmisores de la promesa de la marca y de la cultura de la organización. La relación Empleado/Marca es una relación estratégica que, por motivos incomprensibles, sigue siendo una asignatura pendiente en el desarrollo de las capacidades competitivas de las organizaciones, especialmente en las pymes españolas.

Los departamentos que deberían estar involucrados en la articulación de los programas de internal branding son principalmente el departamento de $\mathrm{RRHH}$, el departamento de marketing y el departamento de operaciones. En caso de contar con un departamento de comunicación, éste debe estar también implicado ya que es el responsable último de que la marca sea percibida de manera homogénea ante todos los públicos.

Los resultados de la investigación realizada sugieren que es necesario evidenciar al sector empresarial y académico la importancia de continuar con el desarrollo conceptual y heurístico del internal branding porque ello redundará en una evolución positiva en las relaciones entre empleados, clientes, marcas y organizaciones. 


\section{Referencias bibliográficas}

AAKER, D. A. (2002). "Building Strong Brands". En: Academy of Management Journal, $n^{\circ} 37(4), p$. 765-802.

AguAdo, M. \& JIMÉNEZ, A. (2009). Employer branding. Córdoba: Almuzara.

Albrecht, S. L. (2010). "Employee engagement: 10 key questions for research and practice. The handbook of employee engagement: Perspective, issues, research and practice" En: ALBRECHT S. L. (2010) Handbook of employee engagement; perpectives, research and practice. Northamptom, MA: Edward Elgar. P. 3-19

BerRY, L. (2000). Cómo descubrir el alma del servicio. Los nueve motores del éxito empresario sostenido. Barcelona: Granica.

CostA, J., (2012). El DirCom hoy: Dirección y gestión de la comunicación en la nueva economía. Barcelona: Costa Punto Com.

Foster, C., PunjaisRi, K., \& Cheng R., (2010), "Exploring the relationship between corporate, internal and employer branding". En: Journal of Product \& Brand Management, Vol. 19, n 6, p. 401 - 409.

GaLINDO, L. J. (1998). Técnicas de investigación en sociedad, cultura y comunicación. Buenos Aires: Eddison Wesley Longman.

GiLLIS T. (2011). The IABC handbook of organizational communications. San Francisco: Joseey Bass.

Grace, D. \& O'cass, A. (2005). "Examining the effects of service brand communications on brand evaluation". En: Journal of Product \& Brand Management, vol. 14, n. ${ }^{\circ}$ 2, p. 106-116.

IND, N. (2001). Living the Brand: How to Transform Every Member of your Organization into a Brand Champion. Londres: Kogan Page.

KeLLER, K. L. (2003). Strategic Brand Management: Building and Managing Brand Equity. Upper Saddle River, NJ: Prentice-Hall.

KING, C. \& GRACE, D. (2008). "Internal branding: Exploring the employee's perspective". En: Journal of Brand Management, vol. 15, n. ${ }^{\circ}$ 5, p. 358-372.

-, (2012). "Examining the antecedents of positive employee brand-related attitudes and behaviours". En: European Journal of Marketing, vol 46(3/4), p. 469-488.

LAMBIN, J. J. (2012). Market driven management: Strategic and operational marketing. London: Palgrave MacMillan.
MAHneRT, K. (2009). The brand Inside. Saarbucken: VDM Verlag.

Mahnert, K. F. \& Torres, A.M. (2007). "The brand inside: The factors of failure and success in internal branding". En: Irish Marketing Review, 19(1), p. 54-63.

MoRTIMER, R. (2002). "Stomach-churning strategies". En: Brand Strategy, vol. 158, abril, p. 20-22.

MaclaVerty, N. \& Oddie, H., (2015). Internal Branding: $A$ how to guide. Toronto: CMA

PunJaIsRi, K., Wilson, A., et al (2009). "Internal branding: an enabler of employees' brandsupporting behaviours". En: Journal of Service Management, Vol. 20 Iss: 2, pp.209 - 226

-, (2011). "Internal branding process: key mechanisms, outcomes and moderating factors". En: European Journal of Marketing, vol. 45(9/10), p.1521-1537.

Ravens, C. (2014). Internal Brand Management in international contexts. Weisbaden: Springer Gabler.

SARTAin, L. \& Schumann, M. (2006). Brand From the Inside: Eight Essentials to Emotionally Connect Your Employees to Your Business. San Francisco, CA: Jossey -Bass.

Schultz, D., et al. (2003). IMC: The next generation: Five steps for delivering value and measuring returns using marketing communication. Boston: McGraw Hill Professional.

Scнміт, B. (2011). Experience Marketing. Boston: Delft.

Thomson, K. \& De Chernatony, L. (1999). "The Buy-in Benchmark: How Staff Understanding and Commitment Impact Brand and Business Performance". En: Journal of Marketing Management 15(8), november, p. 819-835.

WeLCH, M. (2011). "The evolution of the employee engagement concept: communication implications." En: Corporate Communications: An International Journal, 16(4), p. 328-346.

Wimmer, R. D. \& Dominick, J. R. (1996) La investigación científica de los medios de comunicación. Una introducción a sus métodos. Barcelona: Bosch.

ZUCKER, R. (2002). "More than a name - internal branding at Pearl”. En: Strategic Communication Management, 6(4), junio-julio, p. 4-7. 
